subpopulation of these presynaptic receptor sites combines with transmitter to inhibit subsequent release (the presumed negative feedback) and another to promote release (positive feedback). The hypothesis of feedback regulation of transmitter release derives essentially from observations that noradrenaline and some $\alpha$-receptor agonists decrease the stimulation-induced efflux of transmitter and that phenoxybenzamine, yohimbine and some other compounds with $\alpha$-receptor blocking activity increase transmitter release in a variety of test systems. It is supposed that autoregulation of release might function in dopaminergic, cholinergic and other putative transmitter systems as well as in adrenergic nerves. In these latter cases the perineuronal densities of dopamine, acetylcholine and so on are presumed to be monitored during neurotransmission.

The current presynaptic receptor controversy, however, rests on the interpretation of the evidence for and against feedback regulation. Workers in support of it interpret the findings with noradrenaline and yohimbine, for example, to signify actions as agonist and antagonist with a negative-feedback system modulating transmitter release. Recent work from several sources, however, has seriously questioned the validity and feasibility of feedback and criticized the assessment of the effects of agonists and antagonists simply in terms of a neuronal system regulating transmitter release (see the recent debate in Trends Pharmacol. Sci., 1982, Vol. 3, pp. 8-21). The slim volume edited by $\mathbf{J}$. de Belleroche fails regrettably to deal with the current debate except tangentially in the all-toobrief chapter by Drew. In her preface, de Belleroche describes 'negative feedback' and 'feed forward' receptors as if they unquestionably function in the ordinary neurosecretory process. This appears to be far from the case.

The volume itself is a compilation of twelve short articles on diverse and often very specialized aspects of presynaptic receptors. Topics range from a consideration of presynaptic muscarinic receptors in Torpedo electric organ and an immunological approach to acetylcholine receptors in rat synaptosomes to phospholipase neurotoxins in Torpedo. The volume is weighted towards the cholinergic side with five articles; three articles deal primarily with dopamine; leaving 5-hydroxytyramine, GABA and noradrenaline to be dealt with very briefly in one article each. A final short contribution describes some possible electrophysiological mechanisms modulating transmitter release.

The chapters are all adequately done within the space constraints, and some are very good - the piece by Drew on the classification of $\alpha$ receptors is excellent. Overall, however, the book does not succeed completely. It is too limited and specialized in its coverage. Perhaps the subject matter is now too broad and extensive for a small volume such as this to cover well. It does draw attention to some of the activities in the field and in that sense may encourage the reader to

\section{Evolution of the Nervous System,} 2nd Edn

by Harvey B. Sarnat and Martin G. Netsky, Oxford University Press, 1982. 9.95 (xiv + 504 pages) ISBN 0195 027760

The authors have attempted to survey the literature on comparative neuroanatomy and evolution of the vertebrate brain. In this second edition they have greatly expanded the bibliography (from 500 entries in the first edition to the present 1500 ), and have introduced a new chapter on comparative neurochemistry (written by Robert B. Ramsey) and a concluding chapter on brain evolution and behavior, which primarily addresses the origins of human behavior and language. Like its predecessor, the new edition also includes photographs of a series of histological sections of various vertebrate brains. The text is written at a level suitable for graduatelevel courses or for professionals in other fields who want a relatively quick survey of the subject.

The authors state that the study of the evolution of the nervous system represents a major approach to exploring neuroanatomy and they note that, although this approach is one of the most abstract among the neurosciences, such study reveals that 'The functional capabilities of phylogenetically older systems are demonstrated in lower vertebrates, as well as their inadequacies for increasing the range of functions needed by evolving species, including man.' This sentence would seem to imply that the brain in a primitive organism is somehow not a part of that organism and does not adapt and evolve. By definition, "older systems" were capable of 'increasing the range of functions needed by evolving species' or these species would not have evolved! The authors then provide a historical view of an explore this fascinating area. The editor intended that the book might serve as a broad and up-to-date reference text on the subject of presynaptic receptors: It clearly does not meet this goal. The readers will have to wait for a more comprehensive and balanced assessment of the subject matter to fulfil that function.

STANILY KALSNER

Professor, University of Otawa, Health Sciences, Department of Pharmacology, 451 Smyth Road, Ottawa, Ontario, Canada KIH $8 M 5$

increasingly complex vertebrate brain as we are guided up the 'phylogenetic scale' from invertebrates to humans. Thus, once again, comparative neuroanatomy is equated to a mere phylogenetic history of brains, not to elucidating principles of neural organization or to understanding the evolutionary processes responsible for the morphological and functional variation exhibited by modern vertebrate brains.

Part of the problem with this book is that the authors do not appear to be familiar with the important advances in biological thought regarding the way structures are compared, nor do they reflect a contemporary view of evolutionary theory. Instead, the text is riddled with nineteenth-century biological thought. humanistic interpretations, and Scala naturae reflected in the typological characterization of vertebrates as the turtle', 'the frog', etc. This type of thinking ignores, if not denies, biological diversity, since it relegates into a single pattern, or process, numerous species whose neural organization reflects widely varying biological, ecological and behavioral adaptations. Both bony and cartilaginous fish exhibit as much diversity in their neural organization as exists throughout all land vertebrates. So much for 'the shark', not to mention 'the fish'.

The authors do not adequately define the concept of homology, and they clearly do not understand the biological significance of homologous characters. They. state: 'The frequent adaptation of homologous structures to new functions in later species limits the definition of homology to one of structure and excludes function'. Such definition makes it impossible to recognize homologous functions and behaviors and precludes recognition of evolutionary trends in either function or behavior. All comparative physiological and behavioral studies would thus be 
restricted to describing contemporary phenomena.

Equally important, but not surprising, the authors appear to be unaware of independently evolved characters (homoplasy) and thus imply that all similar characters must be homologous, regardless of whether or not they were present in the common ancestor. They repeatedly state that a given character 'first' appears in a particular class of vertebrates; in reality, many of their examples - such as separate, large sensory areas in the pallium of vertebrates, and telencephalic pathways that reach spinal levels - are found only in a few advanced species among different classes of vertebrates and are clearly not homologous but homoplasous, i.e. independently derived.

There are numerous related problems: lack of or inaccurate definitions; incorrect use of morphological descriptors; the authors' concept of the relationship between ontogeny and phylogeny; and their invalid notion that phylogenetically 'new' centers are most susceptible to disease processes. (If both older systems and new centers are inferior, one wonders how organisms manage to function.)

The authors have obviously tried to incorporate an exponentially expanding body of literature into their review and have attempted to supplement older descriptive studies with more recent experimental data. Unfortunately, this has resulted in incompatible and inaccurate statements, as the experimental data frequently refute or implicate the older reports in ways of which the authors are apparently unaware. An additional problem is that many of the brain sections in the photographic atlas are improperly labelled and are of poor quality histologically.

In the last analysis, Sarnat and Netsky can be forgiven much, for they are amateurs in the best sense of the word, and it is obvious that they are deeply interested in synthesizing a field they believe to be important. The greatest value of this synthesis to comparative neurobiologists is, perhaps, as an indication of what other neuroscientists perceive to be our philosophy, our goals, our methods and our achievements. If this book is a valid indication, comparative neurobiologists had better take note.

R. GLENN NORTHCUTT

Professor, Division of Biological Sciences, University of Michigan, Ann Arbor, MI 48109, USA.

\section{Antiepileptic Drug Therapy in Pediatrics}

by Paolo L. Morselli, C. E. Pippenger and J. Kiffin Penny, Raven Press, 1983. $\$ 61.38$ (xii +369 pages) ISBN 0890 048363

There has been a positive explosion over recent years in the number of books appearing on the market on the subject of epilepsy. The advances in monitoring anticonvulsant drugs, the better understanding of pharmacology, and the advent of new therapeutic agents scarcely seem to have justified the phenomenal increase in books related to epilepsy. One epileptologist said recently that he felt there should be a moratorium for two years on any further books appearing unless there was some major advance within the subject. Raven Press themselves have produced a whole series of individual books on different aspects of epilepsy and this book relating to antiepileptic drug therapy in children is, therefore, one of a series.

The book is based upon a symposium, 'Antiepileptic Drugs in Newborn Infants, Children and Adolescents', held in 1981 under the auspices of the International League Against Epilepsy. The book is well produced and easy to read, with no pictorial illustrations other than tables and graphs. The contents cover general aspects, pharmacokinetics, and then a section on the different types of seizure and recommended therapy with a later section on the individual drugs. There are sections on the psychological effects of anticonvulsant drugs and several chapters on basic research and the future.
I thought the first three chapters were good; these are on the developmental aspects of drug kinetics, effects of brain maturation on drug responses, and fatal effects of anticonvulsants. However, they gave a false impression of the rest of the book. There was obviously alot of repetition between the chapters relating to individual seizures and those relating to individual drugs. Some chapters were at a basic, undergraduate level, others were mere repetitions of standard textbook material. It is rather unfortunate that spaced within the book is a vast amount of good material, but the book suffers from being the proceedings of a conference and it is therefore very difficult to identify which chapters one would selectively read. One gets the impression that if the editors had written a good review article at the end, this would have been of much more use since, as it stands, it is difficult to use the book as a reference source. I doubt that many paediatricians would plough

\section{Insect Neurohormones}

by Marie Raabe, Plenum Press, 1982. $\$ 42.50$ (USA and Canada)/\$51.00 (elsewhere) (xiv + 352 pages) ISBN 0306 407825

This is a thorough and timely review of, and introduction to, the endocrinology of insects. It will be particularly useful to the growing numbers of scientists now entering this field, many of whom do not have a background in entomology.

Insect neurobiology, and neuroendocrinology in particular, is currently ex- through the 362 pages as this reviewer did.

The last section raised some very interesting points relating to ethics of paediatric research, animal models for epilepsy, and the effects of hormones on convulsive thresholds.

To summarize, I enjoyed several chapters of the book. 1 felt that there was alot of valuable information spread throughout the pages but that it had all the defects of a book based upon a conference, in that what appears to be a logical series of papers at a conference is not necessarily a sensible series of chapters in a book. The book will be a valuable edition to paediatric libraries, and will be of interest to paediatric neurologists but I doubt that it will find as widespread acceptance amongst general paediatricians as other books on the market.

J. K. BROWN

Consultant Paediatric Neurologist, Royal Hospital for Sick Children, Edinburgh EH ${ }^{9} I L F, U K$.

periencing a rapid expansion. There are two primary reasons for the recent growth of interest in the insect nervous and endocrine systems. First, with the growth of the neurosciences it has become clear that a variety invertebrate preparations, including insect preparations, offer some important advantages for the study of transmitter and hormonal action. For those interested in basic mechanisms of chemical communications, insects offer a wide variety of relatively simple cellular preparations. The second reason is reflected in the growth of interest shown 